\title{
ICME: Bridging Interfaces
}

\author{
G.J. SCHMITZ ${ }^{1,2,3}$ \\ 1.-Access e.V., RWTH Aachen, Intzestr. 5, 52072 Aachen, Germany. 2.-e-mail: g.j.schmitz@ \\ micress.de. 3.—e-mail: g.j.schmitz@access.rwth-aachen.de
}

Integrated computational materials engineering (ICME) is an emerging discipline with a holistic approach. Interfaces play an important role in ICME, such as interfaces between scales, interfaces between materials classes, interfaces between individual phenomena, interfaces between individuals, and interfaces between communities and eventually communication barriers, to name only a few.

This issue of JOM comprises several articles addressing some of these interfaces:

An article on "Cross-Permeability of the Semisolid Region in Directional Solidification" by Böttger et al. bridges the scales between interdendritic and macroscopic melt flow. An article by Shokoohi and Naderi on "Development and Experimental Validation of Morphology Predictive Model for Compatibilized Ternary Polymer Blends" adds polymers as another material class to the ICME community, which by now in large part is strongly focused on metals and alloys and their processing. "Modeling the Electrical Contact Resistance at Steel-Carbon Interfaces" by Brimmo and Hassan tackles the influence of electrical currents on the evolution of materials as an additional phenomenon beyond those occurring in typical processes. "Industrial Needs for ICME" by Konter et al. is a joint contribution of a company, a research center, and a university. Besides bridging industry and academia, this article bridges aspects of the local material behavior and the origin of structural behavior of entire components.

ICME, however, not only bridges technological barriers but also creates bridges between individuals and even between entire communities. ICME requires a holistic view on things. However, a generalist - to the extreme-knows nothing about everything, while a specialist-again to the extreme-knows everything about almost nothing. ICME needs both specialists and generalists and thus by

Georg J. Schmitz is the guest editor for the ICME Committee of the TMS Materials Processing \& Manufacturing Division, and coordinator of the topic ICME: Bridging Interfaces in this issue. its nature is a team effort bridging between individuals. ICME also bridges the interfaces between different communities. This very fruitful interaction of different communities is highlighted in the article on "Software Solutions for ICME" by Schmitz et al. This article summarizes the findings of an international workshop on ICME and provides an outlook on upcoming activities toward further networking the different communities.

When networking different communities, communication problems frequently have to be overcome. An example is the word "object," which for IT people represents a well-defined data structure, while it is understood as a physical object like a component of an assembly or a feature in a microstructure by engineers. Similar confusion is raised by the word "application," which commonly is understood as a software tool by IT people, while engineers would interpret it as the use of a product for a specific purpose. Even within a community, some wordings are used in an inconsistent way. The "concentration" of an element in an alloy in general is given in wt.\%. This unit, however, corresponds to a weight fraction, while the correct unit of concentration would be mass per volume, leading to a confusion with the term "density" then.

Communication standards thus play an important role in ICME not only between people but also between the experimental world, the different model worlds, and respective software tools. The article "Microstructure Modeling in Integrated Computational Materials Engineering (ICME) Settings: Can HDF5 Provide the Basis for an Emerging Standard for Describing Microstructures?" by Schmitz proposes a possible communication standard between different tools in the area of microstructure descriptions.

In summary, ICME not only means bridging interfaces but also crossing boundaries between disciplines and communities and in some sense even creating and defining interfaces, as long as those are standardized and allow for easy interoperability. The jpg/mpg formats for pictures/movies and 
their widespread daily use in society provide a taste of what is possible on the basis of a standardized information exchange.

The following articles being published under the topic of "ICME: Bridging Interfaces" provide excellent details and research on the subject. To download any of the articles, follow the url http://link. springer.com/journal/11837/68/1/page/1 to the table of contents page for the January 2016 issue (vol. 68 , no. 1).

- "Cross-Permeability of the Semisolid Region in Directional Solidification: A Combined PhaseField and Lattice-Boltzmann Simulation Approach" by B. Böttger, C. Haberstroh, and N. Giesselmann

- "Development and Experimental Validation of Morphology Predictive Model for Compatibilized Ternary Polymer Blends I. Effect of Interfacial
Tension" by Shirin Shokoohi and Ghasem Naderi

- "Modeling the Electrical Contact Resistance at Steel-Carbon Interfaces" by Ayoola T. Brimmo and Mohamed Hassan

- "Industrial Needs for ICME" by A.W.A. Konter, H. Farivar, J. Post, and U. Prahl

- "Software Solutions for ICME" by G.J. Schmitz, A. Engstrom, R. Bernhardt, U. Prahl, L. Adam, J. Seyfarth, M. Apel, C. Agelet de Saracibar, P. Korzhavyi, J. Agren, and B. Patzak

- "Microstructure Modeling in Integrated Computational Materials Engineering (ICME) Settings: Can HDF5 Provide the Basis for an Emerging Standard for Describing Microstructures?" G.J. Schmitz 